\title{
Ontogeny of Small Intestinal Drug Transporters and Metabolizing Enzymes Based on Targeted Quantitative Proteomics
}

\author{
Márton Kiss, ${ }^{1}$ Richard Mbasu, ${ }^{1}$ Johan Nicolaï, Karin Barnouin, Apoorva Kotian, Miriam G. \\ Mooij, Nico Kist, Rene M. H. Wijnen, Anna-Lena Ungell, Paul Cutler, Frans G. M. Russel, \\ and Saskia N. de Wildt
}

\begin{abstract}
Department of Pharmacology and Toxicology, Radboud Institute for Health Sciences, Radboud University Medical Center, Nijmegen, The Netherlands (M.K., F.G.M.R., S.N.d.W.); Development Science (R.M., K.B., A.K., P.C.), and Statistical Sciences and Innovation (N.K.), UCB BioPharma, Slough, United Kingdom; Development Science, UCB BioPharma SRL, Braine-l'Alleud, Belgium (J.N., A.-L.U.); Department of Pediatrics, Willem-Alexander Children's Hospital, Leiden University Medical Centre, Leiden, The Netherlands (M.G.M.); and Intensive Care and Department of Pediatric Surgery, Erasmus MC-Sophia Children's Hospital, Rotterdam, The Netherlands (R.M.H.W.)
\end{abstract}

Received June 1, 2021; accepted September 13, 2021

\section{ABSTRACT}

Most drugs are administered to children orally. An information gap remains on the protein abundance of small intestinal drug-metabolizing enzymes (DMEs) and drug transporters (DTs) across the pediatric age range, which hinders precision dosing in children. To explore agerelated differences in DMEs and DTs, surgical leftover intestinal tissues from pediatric and adult jejunum and ileum were collected and analyzed by targeted quantitative proteomics for apical sodium-bile acid transporter, breast cancer resistance protein (BCRP), monocarboxylate transporter 1 (MCT1), multidrug resistance protein 1 (MDR1), multidrug resistance-associated protein (MRP) 2, MRP3, organic anion-transporting polypeptide $2 \mathrm{~B} 1$, organic cation transporter 1, peptide transporter 1 (PEPT1), CYP2C19, CYP3A4, CYP3A5, UDP glucuronosyltransferase (UGT) 1A1, UGT1A10, and UGT2B7. Samples from 58 children (48 ileums, 10 jejunums, age range: 8 weeks to 17 years) and 16 adults (8 ileums, 8 jejunums) were analyzed. When comparing age groups, BCRP, MDR1, PEPT1, and UGT1A1 abundance was significantly higher in adult ileum as compared with the pediatric ileum. Jejunal BCRP, MRP2, UGT1A1, and CYP3A4 abundance was higher in the adults compared with children 0-2 years of age. Examining the data on a continuous age scale showed that PEPT1 and UGT1A1 abundance was significantly higher, whereas MCT1 and UGT2B7 abundance was lower in adult ileum as compared with the pediatric ileum. Our data contribute to the deeper understanding of the ontogeny of small intestinal drug-metabolizing enzymes and drug transporters and shows DME-, DT-, and intestinal location-specific, age-related changes.

\section{SIGNIFICANCE STATEMENT}

This is the first study that describes the ontogeny of small intestinal DTs and DMEs in human using liquid chromatography with tandem mass spectrometry-based targeted quantitative proteomics. The current analysis provides a detailed picture about the maturation of DT and DME abundances in the human jejunum and ileum. The presented results supply age-related DT and DME abundance data for building more accurate PBPK models that serve to support safer and more efficient drug dosing regimens for the pediatric population.

\section{Introduction}

Oral drug delivery in the pediatric population is more challenging than in adults. It is the preferred dosing route for patients of all ages but adds a layer of complexity to the determination of pediatric dosing

This project was financed by UCB Biopharma and the Dutch Ministry of Economic Affairs by means of the PPP Allowance made available by the Top Sector Life Sciences \& Health to stimulate public-private partnerships.

We report that co-authors Richard Mbasu, Johan Nicolaï, Karin Barnouin, Apoorva Kotian, Nico Kist, Anna-Lena Ungell, and Paul Cutler were employees of UCB Biopharma during the course of the study.

${ }^{1}$ M.K. and R.M. contributed equally to this work and shared first authorship. dx.doi.org/10.1124/dmd.121.000559.

S This article has supplemental material available at dmd.aspetjournals.org. regimens that are often extrapolated from adult values based on body weight or surface area (Ritschel, 1980; Batchelor and Marriott, 2015; Sawrey et al., 2019). These methods do not take developmental changes of absorption, distribution, metabolism, and excretion (ADME) pathways into account, resulting in age-related variation in exposure that increase the risk of toxicity or therapy failure (Weiss et al., 1960; Ferro, 2015). Although our understanding of ontogeny of ADME processes has greatly increased over recent years, the ontogeny of intestinal drug transporters (DTs) and drug-metabolizing enzymes (DMEs) remains unresolved. Drug transporters are comprised of ATP-binding cassette (ABC) and solute carrier (SLC) families that reside in the luminal and basolateral plasma membranes of enterocytes. In contrast, drug-metabolizing enzymes are present in the cytosol or localize to the membranes of the endoplasmic reticulum. These protein families play a key role in

ABBREVIATIONS: ABC, ATP-binding cassette; ADME, absorption, distribution, metabolism, and excretion; ASBT, apical sodium-bile acid transporter; BCRP, breast cancer resistance protein; BLQ, below limit of quantitation; DME, drug-metabolizing enzyme; DT, drug transporter; FA, formic acid; FDR, false discovery rate; IHC, immunohistochemistry; LC-MS/MS, liquid chromatography with tandem mass spectrometry; LLOQ, lower limit of quantitation; MCT1, monocarboxylate transporter 1; MDR1, multidrug resistance protein 1; MRP, multidrug resistance-associated protein; OATP2B1, organic anion-transporting polypeptide 2B1; OCT1, organic cation transporter 1; PEPT1, peptide transporter 1; PI, protease inhibitor; PNA, postnatal age; SIL, stable isotope-labeled; SLC, solute carrier; TQP, targeted quantitative proteomics; UGT, UDP glucuronosyltransferase. 
determining oral drug bioavailability (Giacomini et al., 2010; Xie et al., 2016; Zamek-Gliszczynski et al., 2018). Recently, the age-dependent variation in hepatic and renal abundances of DTs has been investigated by our group and others using proteomics (Prasad et al., 2016; Bhatt et al., 2017, 2019; Boberg et al., 2017; van Groen et al., 2018; Cheung et al., 2019; Ladumor et al., 2019; Li et al., 2019). These data imply that the abundance of intestinal DMEs and DTs follow specific developmental patterns. Similarly, for the intestine, available gene expression and semiquantitative protein data indicate DT- and DME-specific variation as a function of age. For example, multidrug resistance protein 1 (MDR1) protein was detected from the 12th week of gestational age by immunohistochemistry (IHC). Interestingly, postnatal MDR1 mRNA expression levels were similar in neonates and adults (Konieczna et al., 2011; Mooij et al., 2014). In contrast, Western blot analysis showed that CYP3A4 protein levels in fetal and neonatal duodenum are significantly lower as compared with other pediatric age groups (Johnson et al., 2001). Unfortunately, the methods used to obtain this information have several limitations. Firstly, IHC is suboptimal for quantitation of protein expression. Secondly, mRNA expression may not reflect protein content and enzymatic activity in intestinal tissue because of translational and post-translational variations (Couto et al., 2019). Finally, Western blot requires significant amounts of tissue to measure protein content, which is especially challenging in the pediatric population, in which tissue is scarce.

Researchers have therefore looked to apply more advanced quantitative approaches, such as targeted quantitative proteomics (TQP), to determine ADME protein levels in animal and adult human intestinal tissues (Kuno et al., 2019; Couto et al., 2020). Unlike Western blot and quantitative polymerase chain reaction, TQP does not require large tissue amounts. Furthermore, TQP allows for multiplexing, making this method a viable technique to maximize the amount of information that can be obtained from pediatric tissue samples that are often small and scarce (Yu et al., 2020).

Based on the available pediatric data on ontogeny of ADME protein levels, we hypothesized that small intestinal DTs and DMEs would show significant age-related, intestinal region-specific, and protein-specific maturation. Using TQP, we aimed to assess the abundance of clinically relevant DTs and DMEs in jejunal and ileal samples across the pediatric age range and compare them to adult values.

\section{Materials and Methods}

Chemicals and Reagents. EDTA, sucrose, histidine, dithiothreitol, iodoacetamide, triethylammonium bicarbonate buffer, formic acid (FA), and trifluoroacetic acid were obtained from Sigma-Aldrich (Poole, UK). RapiGest was obtained from Waters Corporation (Milford, MA). Sequencing grade modified trypsin was purchased from Promega (Madison, WI). Protease inhibitor (PI) (P8340) was obtained from Sigma-Aldrich (Poole, UK). Pierce Rapid Gold BCA assay kit and PBS without $\mathrm{Ca}^{2+}$ and $\mathrm{Mg}^{2+}$ were obtained from Thermo Fisher (Paisley, UK), whereas stable isotope-labeled (SIL) internal standards of analytical grade with a purity $\geq 95 \%$ were purchased from Thermo Fisher (Rockford, IL). CryoPREP (CP02 Covaris) and Tissue Tube TTI were obtained from Covaris (Woburn, MA).

Tissue Samples. Pediatric small intestinal tissues were obtained as leftover material from intestinal surgeries at Erasmus MC Sophia Children's Hospital (Rotterdam, The Netherlands). These jejunal and ileal tissues were assessed for exact anatomic localization by the surgeon. To minimize the risk of tissue degradation, collection was done as follows: All tissues were kept on ice $\left(0^{\circ} \mathrm{C}\right)$ until snap-frozen within 30 minutes in precooled isopentane $\left(-80^{\circ} \mathrm{C}\right)$ except for one sample that was conserved after 90 minutes (Mager et al., 2007). Additionally, leftover ileal tissues were obtained with a similar protocol at Radboud UMC (Nijmegen, Netherlands). These tissues were collected and stored in carbogenated Krebs-Ringer buffer $\left(0^{\circ} \mathrm{C}\right)$ until snap-frozen in liquid nitrogen within 2 hours after excision (Polentarutti et al., 1999). The pediatric tissue collection protocol was evaluated by the Research Ethics Boards of Radboud UMC (CMO number: 2017-3958) and Erasmus MC (MEC 2012-280). As only leftover tissue and electronic health record data were collected, the need for formal Research Ethics Boards approval was waived according to the Dutch Law on Human Research. Written parental and patient-informed consent forms were obtained ahead of the surgical procedures. Detailed characteristics of the pediatric patients are provided in Supplemental Table 1. Because of privacy and anonymization regulations, patient information except age and underlying diagnosis were not available to us. Leftover adult jejunal tissues from Whipple procedures and terminal ileal tissues from (hemi)colectomies were collected anonymously at the Radboud UMC (Nijmegen, The Netherlands). As no patient-related information was accessed, no ethical approval and informed consent was necessary based on the Dutch Law on Human Research. Adult intestinal tissues were handled in the same way as described for the pediatric tissues collected at the Radboud UMC.

Sample Preparation. All procedures were performed with precooled reagents and instruments at $4^{\circ} \mathrm{C}$. Snap-frozen intestinal tissue samples $( \pm 150 \mathrm{mg})$ were crushed using a cryoPREP CP02 (Covaris, Woburn, MA). The crushed tissue powder was suspended in $1 \mathrm{ml}$ of homogenizing buffer (solution $\mathrm{C})(\mathrm{pH} 7.4)$ consisting of PBS, $0.5 \mathrm{mM}$ EDTA, $0.25 \mathrm{M}$ sucrose, $5 \mathrm{mM}$ histidine, and PI cocktail $(0.1 \%$ v/v) (Hatley et al., 2017). The suspended samples were homogenized using a 10-ml Fisherbrand Potter-Elvehjem Homogenizer (Fisher Scientific, Loughborough, UK) with 10 passes $(1250 \mathrm{rpm})$ while keeping the sample on ice. The homogenate was treated with an ultrasonic probe (Qsonica Llc, Newtown, CT) while on ice for two 10-second bursts at $100 \%$ amplitude with a 30 second resting interval. It was then transferred into a 1.5 -ml ultra-centrifuge tube (Beckman Coulter Life Sciences, Indianapolis, IN) and centrifuged at 100,000 × $\mathrm{g}$ using a TLA-55 fixed angle rotor ultracentrifuge (Beckman Coulter Life Sciences, Indianapolis, IN). The supernatant was collected and stored at $-80^{\circ} \mathrm{C}$. The pellet was weighed prior to resuspending in $500 \mu \mathrm{l}$ of solution $\mathrm{C}$ in an Eppendorf tube and then rehomogenized with the ultrasonic probe for two 10-second bursts at 50\% amplitude with a 30 -second resting interval while on ice. The total protein concentration of the postcentrifugation resuspended pellet fraction (crude membrane) was determined with Pierce Rapid Gold BCA assay kit.

Approximately $500 \mu \mathrm{g}$ of the resuspended pellet fraction was taken, and the volumes for each sample were adjusted equally with $10 \mathrm{mM}$ triethylammonium bicarbonate. After adding $10 \mu \mathrm{l}$ of horse myoglobin $(0.4 \mathrm{mg} / \mathrm{ml})$ and $50 \mu \mathrm{l}$ of $0.2 \%$ RapiGest, the samples were reduced with $10 \mathrm{mM}$ dithiothreitol (final concentration) at $60^{\circ} \mathrm{C}$ for 30 minutes and alkylated with $15 \mathrm{mM}$ iodoacetamide (final concentration) in the dark at room temperature for 30 minutes. The samples were then spiked with $6.6 \mathrm{nM}$ (final concentration) SIL internal standard, and proteolytic digestion was performed using sequencing grade modified trypsin (enzyme/substrate ratio: $1: 25$ ) at $37^{\circ} \mathrm{C}$ for 4 hours. After this time, the same amount of sequencing grade modified trypsin was added to the samples and left to incubate for another 12 hours at $37^{\circ} \mathrm{C}$. The reaction was quenched with $0.5 \%$ trifluoroacetic acid (final concentration) and incubated for 45 minutes at $37^{\circ} \mathrm{C}$ to cleave Rapigest. Finally, the digests were centrifuged at $16,000 \times g$ for 15 minutes, and the clear supernatant was collected. The samples were diluted (1:1) with $0.1 \% \mathrm{FA}$, and $50 \mu \mathrm{g}$ of protein was loaded on column for liquid chromatography with tandem mass spectrometry (LC-MS/MS) analysis.

LC-MS/MS Analysis. Multiple reaction monitoring analysis was performed using a 6500 QTRAP (ABSciex UK Limited, Warrington, UK) coupled to a Shimadzu LC (LC-30A Nexera) UHPLC system (Shimadzu, Wood Dale, IL). Fifty microliters of diluted digests was injected into the LC-MS/MS system. Chromatographic separation was achieved by using a reverse phase $\mathrm{C} 18$ column (UPLC PEPTIDE XB-C18 LC column $(100 \times 2.1 \mathrm{~mm}, 1.7 \mu \mathrm{m}$, Phenomenex, Torrance, CA)) and gradient elution at a flow rate of $500 \mu \mathrm{l} / \mathrm{min}$. A linear gradient of $5 \%-40 \% \mathrm{~B}$ (98\% acetonitrile in $0.1 \% \mathrm{FA})$ in 22.5 minutes followed by a 4-minute wash with $100 \% \mathrm{~B}$ was employed. The column oven was maintained at $50^{\circ} \mathrm{C}$, and autosampler was maintained at $8^{\circ} \mathrm{C}$. All experiments were performed in positive electrospray ionization mode. The ion source block temperatures and capillary voltages were kept constant at $500^{\circ} \mathrm{C}$ and $5.5 \mathrm{kV}$, respectively.

The small intestinal transporters and enzymes [ASBT, BCRP, CYP2C19, CYP3A4, CYP3A5, MCT1, MDR1, multidrug resistance-associated protein (MRP) 2, MRP3, organic anion-transporting polypeptide 2B1 (OATP2B1), OCT1, peptide transporter 1 (PEPT1), UDP glucuronosyltransferase (UGT) 1A1, UGT1A10, UGT2B7, and villin-1] were selected based on their clinical relevance and prevalence (Zamek-Gliszczynski et al., 2018). A single multiple reaction monitoring acquisition method was used for all the transporters and 
enzymes (Supplemental Table 2). Initially, SIL peptides were analyzed using a Q-Exactive Plus (ThermoScientific, Bremen, Germany) on data-dependent acquisition mode. Subsequently, Skyline 20.1.0.76 software (University of Washington, Seattle, WA) was used to select the top three high-intensity transitions for each peptide. These transitions were monitored simultaneously for identification and quantitation. Each selected peptide was unique to the protein of interest and followed the in-silico peptide criteria defined by Kamiie et al. (2008). Two peptides per protein were analyzed. Declustering potentials and collision energies were manually optimized for every peptide by infusion tuning. The most intense transition of the peptide was used for quantitation (Gröer et al., 2013; Drozdzik et al., 2018). SIL peptides were used as a single point calibrant for absolute quantification. Prior to sample analysis, calibration curve linearity was checked for each peptide, and based on this analysis, the appropriate amount of SIL was spiked into the sample. Lower limit of quantitation (LLOQ) was also determined during this test (Supplemental Table 2). Peak identification, integration, and quantification were performed using Analyst MultiQuant 3.3 software.

Data Analysis and Statistics. Protein abundance data were measured in $\mathrm{pmol} / \mathrm{mg}$ membrane protein. For samples below limit of quantitation (BLQ) values, data imputation was performed by adding LLOQ/2 (pmol/mg membrane protein) values to the non-normalized dataset to replace BLQ datapoints (Supplemental Table 4). Sample heterogeneity made normalization of the protein abundance values necessary, and villin-1 was selected as a constitutively expressed enterocyte marker (Zhang et al., 2020). Two different normalization calculations were performed depending on the analysis. In the first approach, protein abundance values (pmol/mg membrane protein) were multiplied by the ratio of villin-1 protein abundance in the sample and the average villin-1 protein abundance in the studied tissue (eq. 1; Figs. 1 and 2).

$$
\text { protein abundance in sample } \times \frac{\text { villin }-1 \text { protein abundance in sample }}{\text { average villin }-1 \text { protein abundance }}
$$

In the second approach, protein abundance values (pmol/mg membrane protein) were divided by the villin-1 abundance ( $\mathrm{pmol} / \mathrm{mg}$ membrane protein) and $\log _{2}-$ transformed to normalize the values (eq. 2; Supplemental Table 4; Tables 1 and 2).

$$
\log _{2}\left(\frac{\text { protein abundance in sample }}{\text { villin }-1 \text { protein abundance in sample }}\right)
$$

Relationship between age and protein abundance. Age groups were defined for jejunum (0-2 years, 2-18 years, adults) and ileum (0-2 years, 2-12 years, 12-18 years, adults). To explore differences between age groups, for the villin-1 corrected protein abundances with the imputed values included, the Kruskal-Wallis test was performed. Significance values were adjusted using Bonferroni correction for multiple comparisons among age groups. In the following step, Bonferroni correction for the number of different proteins was implemented by adjusting the significance threshold to $P<0.0033$ (Fig. 1). The same analysis was performed on the non-normalized dataset also (Supplemental Fig. 1).

Additionally, the relationship between postnatal age (PNA) and the villin-1corrected protein abundances was determined by calculating the Spearman's correlation coefficient. False discovery rate (FDR) correction was used to account for the multiple proteins in pediatric ileal samples but not in jejunum due to the low number of samples (Table 1). The analysis was also performed by excluding the imputed values. In addition, the effect of villin-1 correction was independently evaluated for its effect on relative protein abundance levels in the jejunum and the ileum. The same statistical tests were applied on the dataset without normalization. All calculated values are shown in Table 1; only comparisons with an FDR $<0.05$ were considered relevant.

Correlations between proteins in the pediatric ileum were calculated using Spearman's correlation and shown in Table 2 if they had an adjusted $P$ value $<$ 0.000476 after Bonferroni correction. Non-normalized pediatric ileal abundance data were also interrogated (Supplemental Table 3) with the same statistical probe and stringency.

Statistical analyses were performed, and figures were generated with Graphpad Prism version 5.0.3, IBM SPSS Statistics version 25, and R version 4.0.3.

\section{Results}

Tissue Collection. Small intestinal tissue samples from 58 pediatric (jejunum, jejuno-ileal junction, proximal and distal ileum, terminal ileum) and 16 adult patients (proximal jejunum, terminal ileum) were available for the study. At Erasmus MC 36 ileum and 10 jejunum samples from pediatric patients aged between 0 weeks and 16 years were collected. Leftover ileal tissues from 12 patients aged between 8 weeks and 17 years were obtained at Radboud UMC. Detailed description of the pediatric samples regarding patient PNA, gestational age, exact tissue origin, underlying disease, and surgical intervention is summarized in Supplemental Table 1. All adult tissues ( 8 jejunal and 8 ileal samples) were collected at Radboud UMC without any accompanying clinical data.

Protein Expression. In total, $82 \%$ of the samples yielded values above the LLOQ. Protein quantitation in $21 \%$ of pediatric samples and $8 \%$ for adults was BLQ. OATP2B1, MDR1, PEPT1, and villin-1 were detected in all samples. The number of samples BLQ for the other proteins are shown by age group in Supplemental Table 4. High interpatient variability in abundance was detected for most proteins (Figs. 1 and 2; Supplemental Figs. 1-3; Supplemental Table 4). Villin-1 abundance was not significantly different between the age groups.

Relationship of Villin-Normalized Protein Abundance with Age-Analysis by Age Groups. In ileum $(n=56)$, BCRP protein levels were significantly higher in adults $(102.3 \pm 37.19 \mathrm{pmol} / \mathrm{mg})$ than in the 0 - to 2 -year age group $(31.75 \pm 33.84 \mathrm{pmol} / \mathrm{mg} ; P<0.0033)$ and in the 12- to 18 -year age group $(28.72 \pm 17.18 \mathrm{pmol} / \mathrm{mg} ; P<0.0033)$ (Fig. 1$)$. MDR1 was significantly higher in adults $(154.3 \pm 53.78 \mathrm{pmol} / \mathrm{mg})$ than in the 0 - to 2 -year age group $(43.57 \pm 21.76 \mathrm{pmol} / \mathrm{mg} ; P<0.00067)$. PEPT1 protein abundance was elevated in adults $(156.0 \pm 42.51 \mathrm{pmol} /$ $\mathrm{mg}$ ) when compared with the 0 - to 2-year age group $(58.34 \pm 31.73$ $\mathrm{pmol} / \mathrm{mg} ; P<0.00067)$. UGT1A1 protein was higher in adults $(150.3 \pm$ $83.93 \mathrm{pmol} / \mathrm{mg})$ than in the 0 - to 2-year age group $(11.73 \pm 17.91 \mathrm{pmol} /$ mg; $P<0.000067)$. In jejunum $(n=18)$, BCRP protein levels were higher in adults $(92.33 \pm 16.55 \mathrm{pmol} / \mathrm{mg})$ than in the 0 - to 2-year age group $(31.98 \pm 11.11 \mathrm{pmol} / \mathrm{mg} ; P<0.0033)$. MRP2 was significantly higher in adults $(71.45 \pm 24.11 \mathrm{pmol} / \mathrm{mg})$ than in the youngest age group $(15.39 \pm 10.48 \mathrm{pmol} / \mathrm{mg} ; P<0.0033)$. UGT1A1 protein abundance increased with age: the abundance was higher in adults $(441.6 \pm 227.5$ $\mathrm{pmol} / \mathrm{mg})$ than in young infants $(18.41 \pm 13.05 ; P<0.0033)$. CYP3A4 protein levels were elevated in adults $(563.6 \pm 588.1 \mathrm{pmol} / \mathrm{mg})$ compared with the youngest age group $(75.80 \pm 64.05 \mathrm{pmol} / \mathrm{mg} ; P<0.0033)$.

Age Effect on Villin-Normalized Protein Abundance Measured on a Continuous Scale. In the ileum $(n=48)$, a positive correlation with PNA was observed for ASBT, MDR1, PEPT1, and UGT1A1, and a negative correlation was observed for MCT1, CYP2C19, and UGT2B7 (Fig. 2; Table 1). After FDR correction, a positive correlation with PNA remained for PEPT1 and UGT1A1, and a negative correlation remained for MCT1 and UGT2B7. In the jejunum only BCRP increased significantly with PNA in the pediatric jejunum $(n=10)$

Interprotein Abundance Correlations. Correlations between protein abundances in the pediatric ileum $(n=48)$ are summarized in Table 2. In general, DTs and DMEs showed a positive correlation with each other at a significance level of $P<0.000476$, whereas negative correlation was not detected. No correlation was found between BCRP, MRP3, UGT1A1, UGT2B7, CYP3A4, and CYP3A5 and any of the other proteins investigated.

\section{Discussion}

We present from a large cohort of pediatric and adult patients the intestinal protein abundance data of DMEs and DTs that are relevant to intestinal drug metabolism and transport. To our knowledge, this is the first report demonstrating the application of TQP to quantify the DMEs and DTs in the intestine of the pediatric population. With our newly developed TQP method, we were able to quantify all proteins in both 

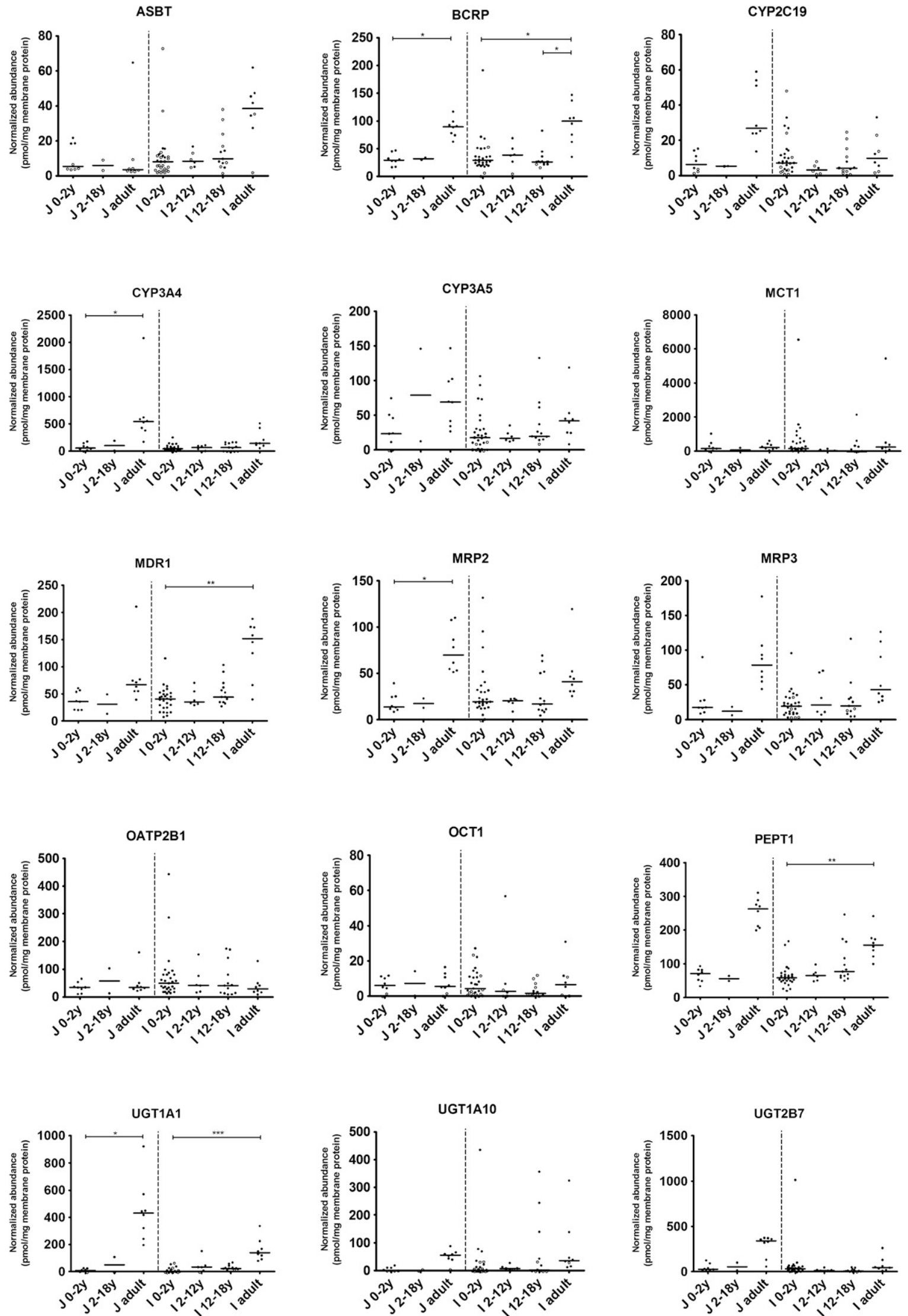

Fig. 1. Relative protein abundances of ABC and SLC transporters, cytochrome P450, and UGT enzymes in the jejunum and ileum of pediatric and adult patients compared among age groups. Data are expressed as pmol/mg membrane protein after normalization for average villin-1 protein abundance (see data analysis section in Methods and Materials). Closed symbols provide a single value determined in singlicate, bars represent medians; empty symbols represent imputed values. Adjusted significance: $* P<0.0033,{ }^{* *} P<0.00067,{ }^{* * *} P<0.000067$ 
ASBT

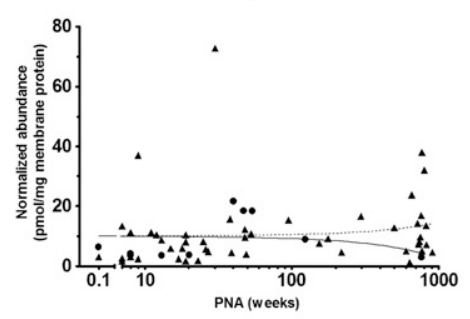

CYP3A4

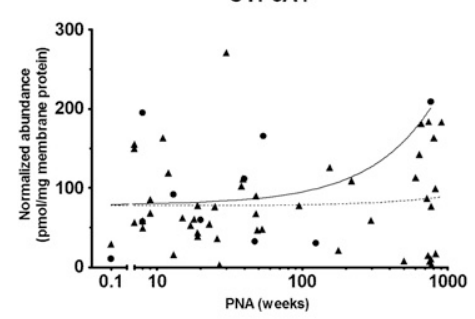

MDR1

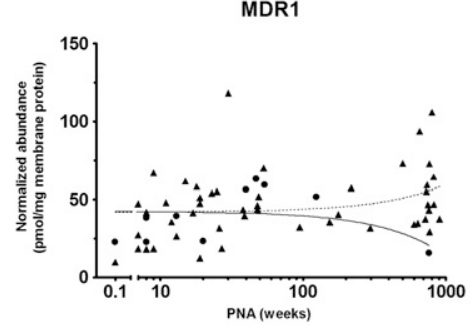

OATP2B1

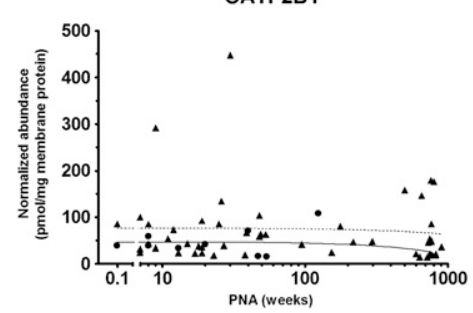

UGT1A1

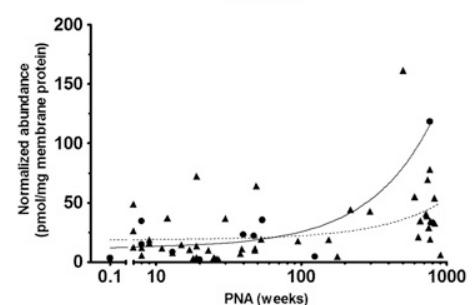

BCRP

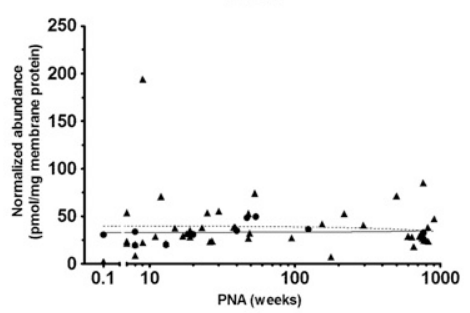

CYP3A5

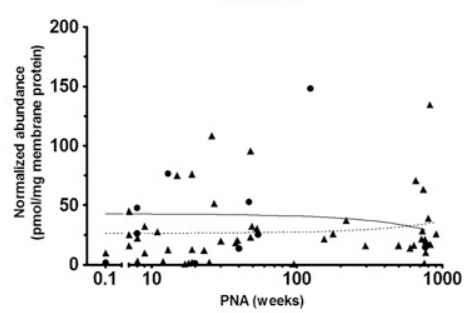

MRP2

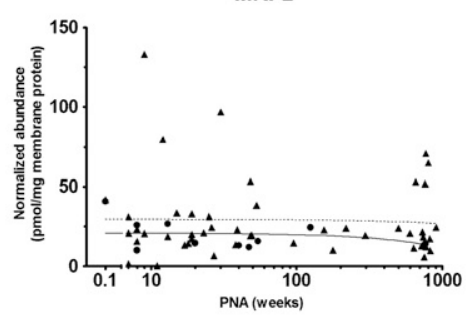

OCT1

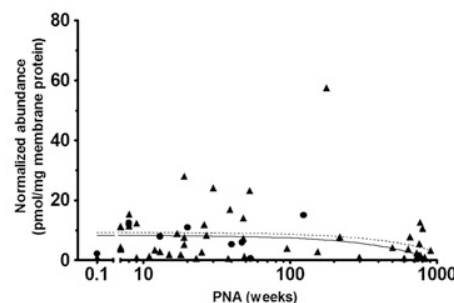

UGT1A10

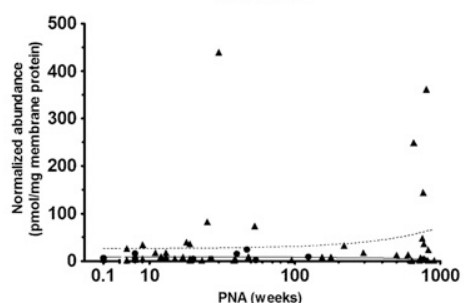

CYP2C19

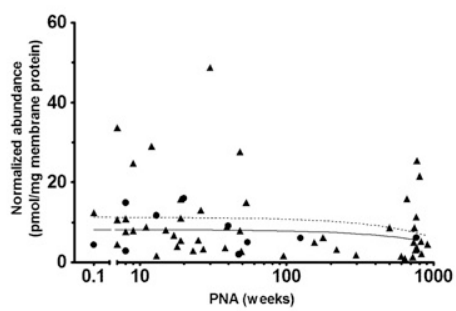

MCT1

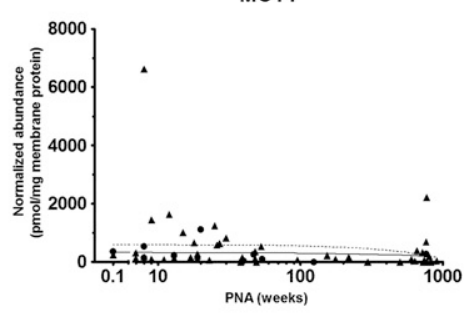

MRP3

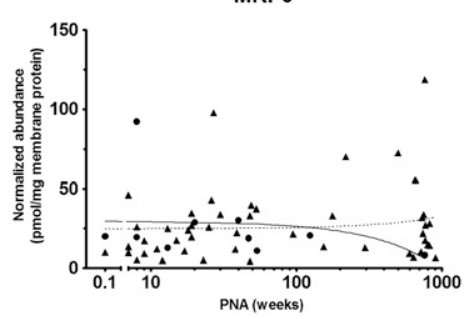

PEPT1

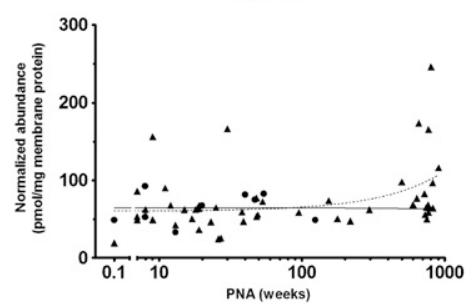

UGT2B7

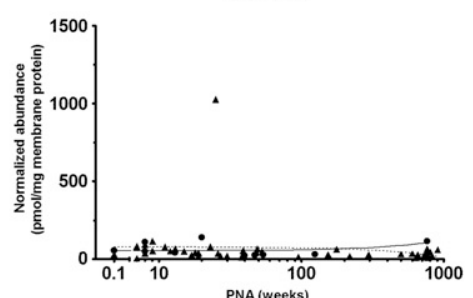

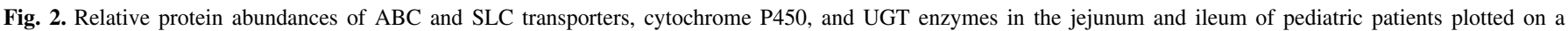

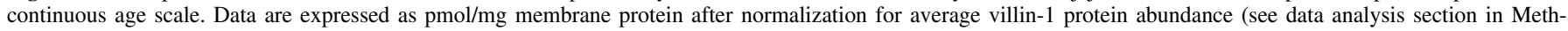
ods and Materials). Lines are linear regressions fitted on the data. Circles and solid line: jejunal samples; triangles and dotted line: ileal samples. 


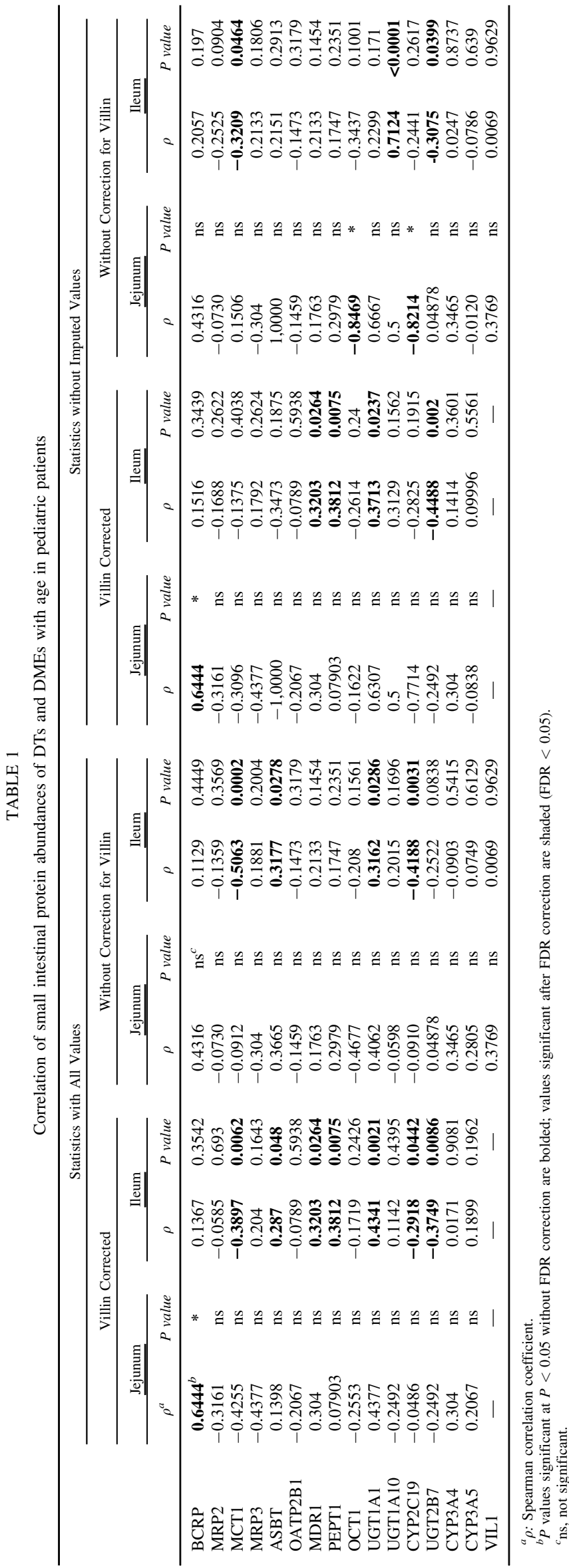

children and adults. Somewhat to our surprise, the impact of age on DME and DT abundance was less evident than we expected based on the limited pediatric data from intestine and the more extensive liver, kidney, and blood-brain barrier data on transporter ontogeny (Mooij et al., 2016; van Groen et al., 2018; Cheung et al., 2019; Verscheijden et al., 2020).

Previously reported intestinal pediatric protein level data originate from IHC or Western blot experiments. Gene expression values do not always predict protein abundance, but for certain proteins strong correlations between age and gene expression were described (Drozdzik et al., 2018, 2019). Indeed, an age-related decrease of OATP2B1 was previously observed at the gene expression level but not in our novel proteomics results when arranged into age groups (Fig. 1) (Mooij et al., 2014). In contrast, our measurements support reverse-transcription polymerase chain reaction analyses indicating that infants express lower quantities of MRP2 compared with adults (Mooij et al., 2014). In our study, BCRP abundance correlates positively with age in both the jejunum and ileum. This finding is in contrast with previous IHC analysis showing no difference between fetal and adult samples (Konieczna et al., 2011). Mooij et al. (2016) detected PEPT1 protein in neonates and infants with decreasing IHC staining intensity toward adolescence, whereas their mRNA data showed a significant increase between the neonatal/infant and the older age groups. Our current findings are in good agreement with the latter-but not with the IHC results - and support that PEPT1 increases with PNA in the ileum (Fig. 1; Table 1). Using Western blotting, Johnson et al. (2001) showed that CYP3A4 protein levels displayed significant differences between fetuses and all other age groups as well as between infants and children above 5 years of age. Similarly, Chen et al. (2015) showed an increase in CYP3A4 after birth up to 11 months PNA with the same technique. Our data indicate a significant increase with age in the jejunum, echoing earlier observations. Studies on MDR1 showed no differences among age groups, neither on the mRNA nor on the protein level after 12 weeks of gestation compared with adults (van Kalken et al., 1992; Fakhoury et al., 2005; Konieczna et al., 2011; Mooij et al., 2014). Interestingly, our age group data shows an age-related increase of MDR1 protein abundance in the ileum (Fig. 1), although significance was not retained after FDR correction on a continuous scale (Table 1). UGT1A1 levels were significantly higher in adults than in the youngest patient group in both intestinal segments tested (Fig. 1) and positively correlated with age in the ileum when measured on a continuous scale (Fig. 2; Table 1). A similar developmental trend was seen in mice expressing humanized UGT1A1 (Fujiwara et al., 2010). Higher protein abundances for UGT1A1 were measured in the adult jejunum and ileum compared with adolescent tissues. This difference was probably due to the administration of irinotecan, a frequently administered agent in adult patients with cancer, which is metabolized by UGT1A1 and can induce its expression (Takahara et al., 2013). Unfortunately, we did not have access to the patient information to check for drug exposure.

UGT2B7 abundance was found to decrease with increasing age (Table 1). This result was unexpected because UGT2B7 is a major enzyme whose absence is responsible for the chloramphenicol-induced Gray baby syndrome in neonates (Kauffman et al., 1981; Chen et al., 2010). Interestingly, this finding suggests organ-dependent ontogeny. Additionally, based on adult UGT2B7 values, less than $1 \%$ of the total enzyme is found in the intestine, whereas the majority is produced in the liver (Drozdzik et al., 2018).

Our study is the first one indicating that MCT1 protein levels may correlate negatively with age in intestines.

In addition, ASBT is normally only present in the ileum; however, in our study it was detected in some of our jejunal samples, whereas in this segment it was BLQ in previous works with one exception (Gröer 
et al., 2013; Drozdzik et al., 2014, 2019; Akazawa et al., 2018). This observation can be attributed either to varying detection limits of the different methods or to external factors. Diet, for instance, can modulate the expression of transporters. For example, the expression of Slc10a in the jejunum has been shown to be increased after oat-containing diet in mice (Andersson et al., 2017). Rats exhibited similar upregulation of Slc10a2/ASBT by $1 \alpha, 25$-dihydroxyvitamin $\mathrm{D}_{3}$ (Chen et al., 2006).

To highlight the developmental changes from birth to adulthood, we have included adult samples in our dataset. Also, adult values allow a comparison of the current dataset with literature values, since this is the first time pediatric intestinal protein abundance levels are reported. The recent article by Couto et al. (2020) provides a summarized overview of most recent publications on TQP of adult human intestinal ADME proteins. All research groups applied their own unique methodology, which makes the comparison of the current dataset to the literature data challenging. When comparing adult protein abundance values from our study with literature values, it becomes obvious that the non-normalized values are higher in comparison with data obtained by other research groups. The underlying reasons can include the fast preservation of the collected tissue samples, the use of the membrane fractionation, application of PI cocktail from the beginning of the sample preparation, thorough sonication, or extensive digestion. Consistency in the digestion was addressed by spiking equal amounts of horse myoglobin into all the samples prior to digestion (Supplemental Fig. 4). Addition of heavy peptides was used also as a quality-control measure to correct for any discrepancies that might occur during sample clean-up and with the LC-MS/MS system.

Protein abundance data in the current study are presented in two ways: 1) relative to villin-1, a constitutively expressed age-independent marker for normalizing for enterocyte levels in intestinal samples, or 2) without normalization. The advantage of normalization is to overcome the issue of uneven sampling in a heterogenous, multilayered tissue like the small intestine. Since in the intestinal tract villin-1 is only expressed in the enterocytes, it is a suitable normalization factor for the number of enterocytes (West et al., 1988). Additionally, it has previously been shown that villin-1 is stable across age groups, including the fetal and neonatal groups (Johnson et al., 2001). Also, according to our own measurements, villin-1 abundance is stable across age groups. However, most published studies describe uncorrected data, and only few research groups applied villin-1 normalization (Vaessen et al., 2017; Zhang et al., 2020). We have chosen to present our data in both ways in an attempt to make cross-comparison with literature results feasible.

A relatively large proportion of pediatric samples had abundance values below the LLOQ. The number of proteins that fall below the LLOQs per tissue correlated with villin-1 abundance (unpublished data). Consequently, LLOQ data may be explained by low villin-1 abundances. Because villin-1 abundance is directly proportional to the number of enterocytes in the sample, we hypothesized that the average yield of all proteins was similarly affected, explaining our finding of relatively frequent protein abundances below the LLOQ, and fortified our assumption that normalization was necessary for the interpretation of the data. We used surrogate values to address the issue and increase statistical power. As we are aware that imputing missing with low values may bias the results, we conducted our statistical analyses with or without including the LLOQ/2 values. Table 1 compares the different outcomes of these calculations. Leaving out BLQ values would be inappropriate because these would then be "missing not at random," which is poison to most statistical methods.

Although no striking differences were found in most protein abundances, it does not mean that there is no age effect on the intestinal metabolism and pharmacokinetics of drugs. There are multiple reasons that back this observation: 1) The effect is influenced/masked by 
medications and food the patients received around the time of sampling. These drugs might change the abundance of DTs and DMEs. Children admitted to hospitals usually have serious health conditions and require medication(s). Disease can also affect DT and DME quantities, but collecting tissues from healthy children is not possible. To reduce the impact of disease we only collected tissue from nondiseased areas. For the stoma tissues, we used the nondiseased areas of the tissue as close to the excision site as possible to avoid using externally exposed tissue. 2) The effect of age is not linear but follows a certain developmental trajectory. This could be solved by including higher number of patients divided into more age groups, but sample collection is a main challenge. 3) Despite our efforts to collect leftover tissue material from all age groups, the neonatal age group was underrepresented in our study population. 4) Genetic polymorphisms may affect gene expression and, ultimately, the function of DTs and DMEs. These investigations would be highly valuable but also introduce new variables. In this experimental setup, drawing proper conclusions would require a larger population. 5) Furthermore, TQP quantitates the protein of interest but gathers no information about its activity. 6) Posttranslational modifications could account for discrepancies when the abundance of a protein does not correlate with its activity. 7) DME and DT activity measurements were out of the scope of the current work, but future studies can bridge this gap in the ontogeny of DTs and DMEs.

The possible implications of our findings include the therapeutic dosing of drugs for pediatric patients. In the current clinical practice, accurate dosing is imperative for agents metabolized by CYP3A4 and UGT1A1, which were found to exhibit age-dependent maturation. Furthermore, BCRP, MDR1, and MRP2 are the main transporters affecting the absorption of the majority of therapeutic substances, thus in the future measuring their abundance could provide the basis for precision drug dosing.

In conclusion, this is the first time that TQP was applied to study the impact of age on DT and DME protein abundance in small intestinal tissues. In spite of the limitations, we showed that our approach detected significant age-related differences for major, clinically relevant DTs and DMEs. The importance of these changes in relation to intestinal drug absorption remains to be demonstrated through the incorporation of the current dataset into physiology-based pharmacokinetic models. To assess the overall importance of the current findings, it will be critical to simultaneously consider all system parameters that are subject to age (e.g., intestinal surface area, length of the intestinal segments). In itself, the lack of change in the abundance of the majority of DTs and DMEs is already a key finding. It can provide better confidence to physicians to prescribe oral drugs and pharmaceutical companies to develop oral dosing regimens for pediatric patients. Future research should expand the dataset by including more neonates to determine possible changes during the first weeks of extrauterine life.

\section{Author Contributions}

Participated in research design: Nicolaï, Barnouin, Kotian, Ungell, Cutler, Russel, de Wildt.

Conducted experiments: Mbasu, Kotian.

Contributed new reagents or analytic tools: Kiss, Mbasu, Nicolaï, Barnouin, Kotian, Mooij, Wijnen.

Performed data analysis: Kiss, Barnouin, Kotian, Kist.

Wrote or contributed to the writing of the manuscript: Kiss, Mbasu, Nicolaï, Barnouin, Kotian, Wijnen, de Wildt.

\section{Acknowledgments}

The invaluable suggestions of Prof. Stefan Oswald regarding the optimization of the TQP method development are highly appreciated.

\section{References}

Akazawa T, Uchida Y, Miyauchi E, Tachikawa M, Ohtsuki S, and Terasaki T (2018) High expression of UGT1A1/1A6 in monkey small intestine: comparison of protein expression levels of cytochromes P450, UDP-glucuronosyltransferases, and transporters in small intestine of cynomolgus monkey and human. Mol Pharm 15:127-140.

Andersson KE, Chawade A, Thuresson N, Rascon A, Öste R, Sterner O, Olsson O, and Hellstrand $P$ (2017) Wholegrain oat diet changes the expression of genes associated with intestinal bile acid transport. Mol Nutr Food Res 61:61.

Batchelor HK and Marriott JF (2015) Formulations for children: problems and solutions. Br J Clin Pharmacol 79:405-418.

Bhatt DK, Gaedigk A, Pearce RE, Leeder JS, and Prasad B (2017) Age-dependent protein abundance of cytosolic alcohol and aldehyde dehydrogenases in human liver. Drug Metab Dispos 45: 1044-1048.

Bhatt DK, Mehrotra A, Gaedigk A, Chapa R, Basit A, Zhang H, Choudhari P, Boberg M, Pearce RE, Gaedigk R, et al. (2019) Age- and genotype-dependent variability in the protein abundance and activity of six major uridine diphosphate-glucuronosyltransferases in human liver. Clin Pharmacol Ther 105:131-141.

Boberg M, Vrana M, Mehrotra A, Pearce RE, Gaedigk A, Bhatt DK, Leeder JS, and Prasad B (2017) Age-dependent absolute abundance of hepatic carboxylesterases (CES1 and CES2) by LC-MS/MS proteomics: application to PBPK modeling of oseltamivir in vivo pharmacokinetics in infants. Drug Metab Dispos 45:216-223.

Chen M, LeDuc B, Kerr S, Howe D, and Williams DA (2010) Identification of human UGT2B7 as the major isoform involved in the O-glucuronidation of chloramphenicol. Drug Metab Dispos 38:368-375.

Chen X, Chen F, Liu S, Glaeser H, Dawson PA, Hofmann AF, Kim RB, Shneider BL, and Pang KS (2006) Transactivation of rat apical sodium-dependent bile acid transporter and increased bile acid transport by 1alpha,25-dihydroxyvitamin D3 via the vitamin D receptor. Mol Pharmacol 69:1913-1923.

Chen YT, Trzoss L, Yang D, and Yan B (2015) Ontogenic expression of human carboxylesterase-2 and cytochrome P450 3A4 in liver and duodenum: postnatal surge and organ-dependent regulation. Toxicology 330:55-61.

Cheung KWK, van Groen BD, Spaans E, van Borselen MD, de Bruijn ACJM, Simons-Oosterhuis Y, Tibboel D, Samsom JN, Verdijk RM, Smeets B, et al. (2019) A comprehensive analysis of ontogeny of renal drug transporters: mRNA analyses, quantitative proteomics, and localization. Clin Pharmacol Ther 106:1083-1092.

Couto N, Al-Majdoub ZM, Achour B, Wright PC, Rostami-Hodjegan A, and Barber J (2019) Quantification of proteins involved in drug metabolism and disposition in the human liver using label-free global proteomics. Mol Pharm 16:632-647.

Couto N, Al-Majdoub ZM, Gibson S, Davies PJ, Achour B, Harwood MD, Carlson G, Barber J, Rostami-Hodjegan A, and Warhurst G (2020) Quantitative proteomics of clinically relevant drug-metabolizing enzymes and drug transporters and their intercorrelations in the human small intestine. Drug Metab Dispos 48:245-254.

Drozdzik M, Busch D, Lapczuk J, Müller J, Ostrowski M, Kurzawski M, and Oswald S (2018) Protein abundance of clinically relevant drug-metabolizing enzymes in the human liver and intestine: a comparative analysis in paired tissue specimens. Clin Pharmacol Ther 104:515-524.

Drozdzik M, Busch D, Lapczuk J, Müller J, Ostrowski M, Kurzawski M, and Oswald S (2019) Protein abundance of clinically relevant drug transporters in the human liver and intestine: a comparative analysis in paired tissue specimens. Clin Pharmacol Ther 105:1204-1212.

Drozdzik M, Gröer C, Penski J, Lapczuk J, Ostrowski M, Lai Y, Prasad B, Unadkat JD, Siegmund W, and Oswald S (2014) Protein abundance of clinically relevant multidrug transporters along the entire length of the human intestine. Mol Pharm 11:3547-3555.

Fakhoury M, Litalien C, Medard Y, Cavé H, Ezzahir N, Peuchmaur M, and Jacqz-Aigrain E (2005) Localization and mRNA expression of CYP3A and P-glycoprotein in human duodenum as a function of age. Drug Metab Dispos 33:1603-1607.

Ferro A (2015) Paediatric prescribing: why children are not small adults. Br J Clin Pharmacol 79:351-353.

Fujiwara R, Nguyen N, Chen S, and Tukey RH (2010) Developmental hyperbilirubinemia and CNS toxicity in mice humanized with the UDP glucuronosyltransferase 1 (UGT1) locus. Proc Natl Acad Sci USA 107:5024-5029.

Gröer C, Brück S, Lai Y, Paulick A, Busemann A, Heidecke CD, Siegmund W, and Oswald S (2013) LC-MS/MS-based quantification of clinically relevant intestinal uptake and efflux transporter proteins. J Pharm Biomed Anal 85:253-261.

Hatley OJD, Jones CR, Galetin A, and Rostami-Hodjegan A (2017) Optimization of intestinal microsomal preparation in the rat: a systematic approach to assess the influence of various methodologies on metabolic activity and scaling factors. Biopharm Drug Dispos 38:187-208.

Giacomini KM, Huang SM, Tweedie DJ, Benet LZ, Brouwer KL, Chu X, Dahlin A, Evers R, Fischer V, Hillgren KM, et al.; International Transporter Consortium (2010) Membrane transporters in drug development. Nat Rev Drug Discov 9:215-236.

Johnson TN, Tanner MS, Taylor CJ, and Tucker GT (2001) Enterocytic CYP3A4 in a paediatric population: developmental changes and the effect of coeliac disease and cystic fibrosis. $\mathrm{Br} \mathrm{J}$ Clin Pharmacol 51:451-460.

Kamiie J, Ohtsuki S, Iwase R, Ohmine K, Katsukura Y, Yanai K, Sekine Y, Uchida Y, Ito S, and Terasaki T (2008) Quantitative atlas of membrane transporter proteins: development and application of a highly sensitive simultaneous LC/MS/MS method combined with novel in-silico peptide selection criteria. Pharm Res 25:1469-1483.

Kauffman RE, Miceli JN, Strebel L, Buckley JA, Done AK, and Dajani AS (1981) Pharmacokinetics of chloramphenicol and chloramphenicol succinate in infants and children. J Pediatr 98: $315-320$.

Konieczna A, Erdösová B, Lichnovská R, Jandl M, Cížková K, and Ehrmann J (2011) Differential expression of ABC transporters (MDR1, MRP1, BCRP) in developing human embryos. $J \mathrm{Mol}$ Histol 42:567-574.

Kuno T, Hirayama-Kurogi M, Ito S, and Ohtsuki S (2019) Proteomic analysis of small intestinal epithelial cells in antibiotic-treated mice: changes in drug transporters and metabolizing enzymes. Drug Metab Pharmacokinet 34:159-162.

Ladumor MK, Bhatt DK, Gaedigk A, Sharma S, Thakur A, Pearce RE, Leeder JS, Bolger MB, Singh S, and Prasad B (2019) Ontogeny of hepatic sulfotransferases and prediction of agedependent fractional contribution of sulfation in acetaminophen metabolism. Drug Metab Dispos 47:818-831. 
Li CY, Hosey-Cojocari C, Basit A, Unadkat JD, Leeder JS, and Prasad B (2019) Optimized renal transporter quantification by using aquaporin 1 and aquaporin 2 as anatomical markers: application in characterizing the ontogeny of renal transporters and its correlation with hepatic transporters in paired human samples. AAPS J 21:88

Mager SR, Oomen MH, Morente MM, Ratcliffe C, Knox K, Kerr DJ, Pezzella F, and Riegman PH (2007) Standard operating procedure for the collection of fresh frozen tissue samples. Eur J Cancer 43:828-834.

Mooij MG, de Koning BE, Lindenbergh-Kortleve DJ, Simons-Oosterhuis Y, van Groen BD, Tibboel D, Samsom JN, and de Wildt SN (2016) Human intestinal PEPT1 transporter expression and localization in preterm and term infants. Drug Metab Dispos 44:1014-1019.

Mooij MG, Schwarz UI, de Koning BA, Leeder JS, Gaedigk R, Samsom JN, Spaans E, van Goudoever JB, Tibboel D, Kim RB, et al. (2014) Ontogeny of human hepatic and intestinal transporter gene expression during childhood: age matters. Drug Metab Dispos 42:1268-1274.

Polentarutti BI, Peterson AL, Sjöberg AK, Anderberg EK, Utter LM, and Ungell AL (1999) Evaluation of viability of excised rat intestinal segments in the Ussing chamber: investigation of morphology, electrical parameters, and permeability characteristics. Pharm Res 16:446-454

Prasad B, Gaedigk A, Vrana M, Gaedigk R, Leeder JS, Salphati L, Chu X, Xiao G, Hop C, Evers R, et al. (2016) Ontogeny of hepatic drug transporters as quantified by LC-MS/MS proteomics. Clin Pharmacol Ther 100:362-370.

Ritschel WA (1980) Handbook of Basic Pharmacokinetics, Drug Intelligence Publications, Hamilton, IL 62341

Sawrey EL, Subramanian MW, Ramirez KA, Snyder BS, Logston BB, and Russell GB (2019) Use of body surface area for dosing of vancomycin. J Pediatr Pharmacol Ther 24:296-303.

Takahara N, Nakai Y, Isayama H, Sasaki T, Satoh Y, Takai D, Hamada T, Uchino R, Mizuno S, Miyabayashi K, et al. (2013) Uridine diphosphate glucuronosyl transferase 1 family polypeptide A1 gene (UGT1A1) polymorphisms are associated with toxicity and efficacy in irinotecan monotherapy for refractory pancreatic cancer. Cancer Chemother Pharmacol 71:85-92.

Vaessen SF, van Lipzig MM, Pieters RH, Krul CA, Wortelboer HM, and van de Steeg E (2017) Regional expression levels of drug transporters and metabolizing enzymes along the pig and human intestinal tract and comparison with Caco-2 cells. Drug Metab Dispos 45:353-360. van Groen BD, van de Steeg E, Mooij MG, van Lipzig MMH, de Koning BAE, Verdijk RM, Wortelboer HM, Gaedigk R, Bi C, Leeder JS, et al. (2018) Proteomics of human liver membrane transporters: a focus on fetuses and newborn infants. Eur J Pharm Sci 124:217-227.

van Kalken CK, Giaccone G, van der Valk P, Kuiper CM, Hadisaputro MM, Bosma SA, Scheper RJ, Meijer CJ, and Pinedo HM (1992) Multidrug resistance gene (P-glycoprotein) expression in the human fetus. Am J Pathol 141:1063-1072.

Verscheijden LFM, van Hattem AC, Pertijs JCLM, de Jongh CA, Verdijk RM, Smeets B, Koenderink JB, Russel FGM, and de Wildt SN (2020) Developmental patterns in human blood-brain barrier and blood-cerebrospinal fluid barrier $\mathrm{ABC}$ drug transporter expression. Histochem Cell Biol 154:265-273.

Weiss CF, Glazko AJ, and Weston JK (1960) Chloramphenicol in the newborn infant. A physiologic explanation of its toxicity when given in excessive doses. N Engl J Med 262:787-794.

West AB, Isaac CA, Carboni JM, Morrow JS, Mooseker MS, and Barwick KW (1988) Localization of villin, a cytoskeletal protein specific to microvilli, in human ileum and colon and in colonic neoplasms. Gastroenterology 94:343-352.

Xie F, Ding X, and Zhang QY (2016) An update on the role of intestinal cytochrome P450 enzymes in drug disposition. Acta Pharm Sin B 6:374-383.

Yu Q, Xiao H, Jedrychowski MP, Schweppe DK, Navarrete-Perea J, Knott J, Rogers J, Chouchani ET, and Gygi SP (2020) Sample multiplexing for targeted pathway proteomics in aging mice. Proc Natl Acad Sci USA 117:9723-9732.

Zamek-Gliszczynski MJ, Taub ME, Chothe PP, Chu X, Giacomini KM, Kim RB, Ray AS, Stocker SL, Unadkat JD, Wittwer MB, et al.; International Transporter Consortium (2018) Transporters in drug development: 2018 ITC recommendations for transporters of emerging clinical importance. Clin Pharmacol Ther 104:890-899.

Zhang H, Wolford C, Basit A, Li AP, Fan PW, Murray BP, Takahashi RH, Khojasteh SC, Smith BJ, Thummel KE, et al. (2020) Regional proteomic quantification of clinically relevant noncytochrome P450 enzymes along the human small intestine. Drug Metab Dispos 48:528-536.

Address correspondence to: Dr. Saskia N. de Wildt, Geert Grooteplein 21, Nijmegen, 6525 EZ, The Netherlands. E-mail: saskia.dewildt@radboudumc.nl 